\title{
Temporal contribution to gravitational WKB-like calculations
}

\author{
Valeria Akhmedova ${ }^{\mathrm{a}}$, Terry Pilling ${ }^{\mathrm{b}}$, Andrea de Gill ${ }^{\mathrm{c}}$, Douglas Singleton ${ }^{\mathrm{c}, *}$ \\ a ITEP, B. Cheremushkinskaya 25, Moscow 117218, Russia \\ b Department of Physics, North Dakota State University, Fargo, ND 58105, USA \\ c Physics Department, CSU Fresno, Fresno, CA 93740-8031, USA
}

\section{A R T I C L E I N F O}

\section{Article history:}

Received 27 May 2008

Accepted 7 July 2008

Available online 12 July 2008

Editor: M. Cvetič

\begin{abstract}
A B S T R A C T
Recently, it has been shown that the radiation arising from quantum fields placed in a gravitational background (e.g. Hawking radiation) can be derived using a quasi-classical calculation. Here we show that this method has a previously overlooked temporal contribution to the quasi-classical amplitude. The source of this temporal contribution lies in different character of time in general relativity versus quantum mechanics. Only when one takes into account this temporal contribution does one obtain the canonical temperature for the radiation. Although in this Letter the specific example of radiation in de Sitter space-time is used, the temporal contribution is a general contribution to the radiation given off by any gravitational background where the time coordinate changes its signature upon crossing a horizon. Thus, the quasi-classical method for gravitational backgrounds contains subtleties not found in the usual quantum mechanical tunneling problem.
\end{abstract}

(C) 2008 Elsevier B.V. All rights reserved.

\section{Introduction}

One of the consequences of placing quantum fields in a gravitational background is that these backgrounds can emit radiation. Examples include Hawking radiation [1] for black holes, Unruh radiation [2] for an accelerated observer, and Gibbons-Hawking radiation [3] for an observer in de Sitter space. In each of these cases the spectrum of the radiation is thermal or Planckian. Also each of these space-times has at least one horizon which separates the space-time into different sections.

There are different methods of calculating the radiation from a gravitational background. One method uses the fact that the annihilation/creation operators for some quantum field are in general different in the different space-time sections. By finding the Bogolubov transformation between the different sets of annihilation/creation operators, the spectrum of the radiation emitted can be obtained. Another method uses Wightman functions to study the response of a detector to a given gravitational background [4] (see as well [5]).

In [6,7] a method was developed where the Hawking radiation from a Schwarzschild black hole was obtained using a quasiclassical method. (However see [8] for an earlier quasi-classical calculation of Gibbons-Hawking radiation for de Sitter space-time.) The quasi-classical amplitude is given by the exponent of the imaginary part of the classical action for the particles coming from

\footnotetext{
* Corresponding author.

E-mail addresses: lera@itep.ru (V. Akhmedova), terry.pilling@ndsu.edu (T. Pilling), aadegill@csufresno.edu (A. de Gill), dougs@csufresno.edu (D. Singleton).
}

the near horizon region. There are many advantages to this quasiclassical calculation: (i) The calculations are simple; (ii) One can apply this method to a host of gravitational backgrounds and different spin particles; (iii) The calculation gives a microscopic picture of the radiation; (iv) The back-reaction of the radiation on the black hole metric may be taken into account [7]; (v) This method may also be used to obtain the standard relationship between the black hole temperature and entropy [9].

However, the interpretation of the imaginary contribution to the particle's action as an indication of tunneling has some subtleties. First, if the pair is created behind the horizon neither of the particles can tunnel through the horizon, because the tunneling process in quantum mechanics is described via the solution of a Cauchy problem and has to be causal, while passing through the horizon is acausal. In quantum mechanics the vacuum remains unchanged which is the reason why we can safely convert a time evolution problem into an eigen-value problem. Second, if the pair is created outside a horizon the time for one of the particles to cross the horizon is infinite for the stationary distant observer. However, this same observer should see the radiation from the black hole in finite time after the collapse. In [7] the above subtleties were addressed by taking the horizon to shrink during the pair creation process so that the radiated particles appeared already outside the horizon.

In this Letter the quasi-classical picture is applied to de Sitter space-time using the Hamilton-Jacobi equations. Using the Hamilton-Jacobi equations to find the imaginary contribution to the classical action in a gravitational background is analogous to the Trace-Log calculations in quantum field theory, e.g. 
finding the probability of vacuum decay in an external electromagnetic field [10]. In a Trace-Log calculation one would look for the imaginary contribution to the vacuum decay amplitude, i.e. $\operatorname{Tr} \log \left[\square(g)+m^{2}\right]=\int D x(t) e^{-\frac{i}{\hbar} S(g, x)} \cdot \square(g)$ is the d'Alembertian operator in the background metric $g_{\mu \nu}$ for a particle of mass $m$. On the right-hand side of the equation is a path integral over closed paths and $S$ is the action for particles in the gravitational field. In the quasi-classical approximation $(\hbar \rightarrow 0)$ the saddle-point approximation for the path integral is used. The imaginary contribution comes from the closed paths which cross the horizon going out and back. Similarly in quasi-classics with the Hamilton-Jacobi equations, the imaginary contribution is found from an integral which spans the horizon.

\section{Hamilton-Jacobi equations}

For a scalar field, $\phi(x) \propto \exp \left\{-\frac{i}{\hbar} S(x)+\cdots\right\}$, of mass $m$, the Hamilton-Jacobi equations (to 0th order in $\hbar$ ) are

$g^{\mu \nu}\left(\partial_{\mu} S\right)\left(\partial_{\nu} S\right)+m^{2}=0$.

$S(x)$ is the action of the scalar field and $g^{\mu \nu}$ is the metric of the background space-time.

For stationary space-times with a time-like Killing vector the action can be expressed as the sum of the time and spatial parts

$S\left(x^{\mu}\right)=E t+S_{0}(\vec{x})$,

where $E$ is the particle energy and $x^{\mu}=(t, \vec{x})$. Inserting some particular metric into (1) gives an equation for $S_{0}(\vec{x})$ which has the solution $S_{0}=\int p_{r} d r$ where $p_{r}$ is the canonical momentum for the metric. If $S_{0}$ has an imaginary part the temperature of the radiation can be obtained as follows: the decay rate due to the quasi-classical

$$
\begin{aligned}
\Gamma & \propto \exp \left[-\operatorname{Im} \oint p_{r} d r / \hbar\right] \\
& =\exp \left[-\operatorname{Im}\left(\int p_{r}^{\text {Out }} d r-\int p_{r}^{\text {In }} d r\right) / \hbar\right] .
\end{aligned}
$$

The closed path of integration in the first expression goes across the barrier (i.e. the horizon) and back. The temperature of the radiation is obtained by associating the expression in (3) with a Boltzmann factor $\Gamma \propto \exp [-E / T]$ which gives $T=\frac{\hbar E}{\operatorname{Im} \oint p_{r} d r}$ (note that $T$ is independent of $E$ and we have set $k_{B}=1$ ).

Sometimes the decay rate in (3) is written as $\Gamma \propto$ $\exp \left[ \pm 2 \operatorname{Im} \int p_{r}^{\text {Out, In }} d r / \hbar\right]$ since in many cases $p_{r}^{\text {Out }}=-p_{r}^{\text {In }}$, i.e. crossing the horizon left to right versus right to left only differs by a sign. However, it has been shown [12] that $\exp \left[ \pm 2 \operatorname{Im} \int p_{r}^{\text {Out, In }} d r / \hbar\right]$ is not invariant under canonical transformation but that $\oint p_{r} d r$ is invariant. Thus, only $\oint p_{r} d r$ is a proper observable and only $\oint p_{r} d r$ should be used in (3). In the standard quantum mechanical tunneling problem it does not matter whether one uses $\oint p_{r} d r$ or $\exp \left[ \pm 2 \operatorname{Im} \int p_{r}^{\text {Out, In }} d r / \hbar\right]$ in the exponent of (3) since the tunneling is the same independent of the direction in which one crosses the barrier, i.e. $p_{r}^{\text {Out }}=-p_{r}^{\text {In }}$. In the gravitational quasi-classical problem there are cases in which the passing across the horizon does depend on the direction of traversal, i.e. $p_{r}^{\text {Out }} \neq-p_{r}^{\text {In }}$. An example of this occurs for the black hole solution in Painlevé coordinates [11].

\section{Spatial contribution}

Using the de Sitter space-time the "spatial" contribution to $\Gamma$ is calculated, i.e. the contribution coming from the imaginary part of $S_{0}(\vec{x})$. The full 4D de Sitter space-time can be seen as a hyperboloid $-z_{0}^{2}+z_{1}^{2}+z_{2}^{2}+z_{3}^{2}+z_{4}^{2}=\alpha^{2}$ embedded in 5D Minkowski space-time $d s^{2}=-d z_{0}^{2}+d z_{1}^{2}+d z_{2}^{2}+d z_{3}^{2}+d z_{4}^{2}$. Transforming to static coordinates

$$
\begin{aligned}
& z_{0}=\left(\alpha^{2}-r^{2}\right)^{1 / 2} \sinh (t / \alpha), \quad z_{1}=\left(\alpha^{2}-r^{2}\right)^{1 / 2} \cosh (t / \alpha), \\
& z_{2}=r \sin \theta \cos \phi, \quad z_{3}=r \sin \theta \sin \phi, \quad z_{4}=r \cos \theta,
\end{aligned}
$$

the de Sitter space-time takes the Schwarzschild-like form

$$
d s^{2}=-\left(1-\frac{r^{2}}{\alpha^{2}}\right) d t^{2}+\frac{d r^{2}}{\left(1-\frac{r^{2}}{\alpha^{2}}\right)}+r^{2}\left(d \theta^{2}+\sin ^{2} \theta d \phi^{2}\right) .
$$

This form of the de Sitter metric has a coordinate singularity at $r=\alpha$ which is the event horizon.

Using metric (5) in (1) the following solution for $S_{0}$ is found

$$
\begin{aligned}
S_{0}^{\text {In, Out }} & = \pm \int_{0}^{+\infty} \frac{\sqrt{E^{2}-m^{2}\left(1-\frac{r^{2}}{\alpha^{2}}\right)}}{1-\frac{r^{2}}{\alpha^{2}}} d r \\
& = \pm \int_{0}^{+\infty} \frac{\alpha^{2} \sqrt{E^{2}-m^{2}\left(1-\frac{r^{2}}{\alpha^{2}}\right)}}{(\alpha-r)(\alpha+r)} d r .
\end{aligned}
$$

Because of spherical symmetry the angular part can be neglected. The $+(-)$ sign correspond to different traversal directions across the horizon at $r=\alpha$, i.e. $+=$ In and $-=$ Out. There is a pole on the $r$-axis at $r=\alpha$. One can evaluate the imaginary part of (6) using a semi-circular contour. The result is $S_{0}^{\text {In,Out }}= \pm \frac{i \pi E \alpha}{2}$. The total contribution coming from the spatial part of the action (i.e. from $S_{0}$ ) is obtained by using the closed path which goes out and back across the horizon. The difference in the sign of $S_{0}^{\text {In,Out }}$ is then compensated by a reversal of the integration path with the result that the exponent in (3) is $\oint p_{r} d r=i \pi E \alpha$. This yields a temperature of $T=\frac{\hbar}{\pi \alpha}$ which is twice the Gibbons-Hawking temperature. This factor of two greater temperature was already noticed in [8]. One might suspect that the static coordinates in (4) are "bad" since they do not cover the entire de Sitter space-time, and that one should use another coordinate system which does cover the entire space-time. However since $\oint p_{r} d r$ is invariant under canonical transformations this result will remain the same in any other coordinate system related to (5) by a canonical transformation.

Below it is shown that this disagreement comes from a missed contribution from the temporal part of the total action, $S\left(x^{\mu}\right)$.

\section{Temporal contribution}

The static coordinates (4) cover only the right quadrant of the Penrose diagram of the de Sitter space-time. On crossing the horizon at $r=\alpha$ the coordinates $z_{0}$ and $z_{1}$ reverse their timelike/space-like character. Thus, $z_{0} \propto \cosh (t / \alpha)$ and $z_{1} \propto \sinh (t / \alpha)$. Also now $r>\alpha$ so the order under the square root should be reversed. Therefore the left quadrant of the Penrose diagram of the de Sitter space-time is covered by the following coordinates

$$
\begin{aligned}
& z_{0}=\left(r^{2}-\alpha^{2}\right)^{1 / 2} \cosh (t / \alpha), \quad z_{1}=\left(r^{2}-\alpha^{2}\right)^{1 / 2} \sinh (t / \alpha), \\
& z_{2}=r \sin \theta \cos \phi, \quad z_{3}=r \sin \theta \sin \phi, \quad z_{4}=r \cos \theta .
\end{aligned}
$$

The coordinates in (4) are related to those in (7) simply by letting $t \rightarrow t-i \frac{\pi \alpha}{2}$ in (4). Under this transformation $\sinh (t / \alpha) \rightarrow$ $-i \cosh (t / \alpha)$ and $\cosh (t / \alpha) \rightarrow-i \sinh (t / \alpha)$. The $-i$ is taken care of by an $i$ coming from the square root part. Thus, on crossing the horizon there is a temporal contribution to the imaginary part of the total action, $S\left(x_{\mu}\right)$, given by $\operatorname{Im}\left(E \Delta t^{\operatorname{In}, \text { Out }}\right)=-\frac{\pi \alpha E}{2}$ where the signs correspond to ingoing $(\mathrm{In}=+)$ versus outgoing particles (Out $=-$ ).

Thus, the total imaginary part of the full action (i.e. $S\left(x^{\mu}\right)=$ $\left.E t+S_{0}(\vec{x})\right)$ has equal magnitude contributions from the time and 
spatial parts. Explicitly (remembering that $S_{0}=-\int p_{r} d r$ ) we have $\operatorname{Im}\left(S\left(x^{\mu}\right)=\operatorname{Im}\left(E \delta^{\text {Out }}+E \delta^{\operatorname{In}}-\oint p_{r} d r\right)=-\frac{\pi \alpha E}{2}-\frac{\pi \alpha E}{2}-\pi \alpha E=\right.$ $-2 \pi \alpha E$. This yields the canonical Gibbons-Hawking temperature of $T_{\mathrm{GH}}=\frac{\hbar}{2 \pi \alpha}$.

\section{Conclusion}

Using de Sitter space-time it has been shown that the quasiclassical calculations have an additional subtle feature relative to the standard quantum mechanical tunneling problem. In addition to the spatial contribution to the quasi-classical amplitude (i.e. $\left.\operatorname{Im}\left(S_{0}\right)\right)$ we have shown that there is an equal contribution coming from the temporal part (i.e. $\operatorname{Im}(E \Delta t))$. This is not unexpected since time is treated differently in general relativity and quantum mechanics. In the former it is a dynamical coordinate like the spatial coordinates; in the latter it is a parameter which is distinct from the spatial coordinates. Although the specific case of de Sitter space-time was studied, the same temporal contribution will occur when one crosses a horizon where the time and spatial coordinates switch their time-like/space-like character upon crossing the horizon. E.g. similar temporal contribution appears in the Schwarzschild space-time which exactly fixes the factor of two problem in the Hawking temperature remarked on in [11] (see [13]). Other recent work dealing with the Hawking-Gibbons radiation of de Sitter space-time via tunneling can be found in [14].

Rindler space-time and the associated Unruh radiation provides an example where one has a horizon but does not have a temporal contribution to the quasi-classical amplitude. Rindler space-time for an acceleration $a$ is given by

$d s^{2}=-(1+a x)^{2} d t^{2}+d x^{2}+d y^{2}+d z^{2}$.
One can see that the $t$ coordinate remains time-like on crossing the horizon at $x=-1 / a$. Thus Unruh radiation does not receive a temporal contribution and one obtains the canonical Unruh temperature from only the spatial contribution to the quasi-classical amplitude (see the second reference in [11]).

\section{Acknowledgements}

Authors would like to thank E.T. Akhmedov for valuable discussions. V.A. would like acknowledges support from the Agency of Atomic Energy of Russian Federation.

\section{References}

[1] S.W. Hawking, Commun. Math. Phys. 43 (1975) 199.

[2] W.G. Unruh, Phys. Rev. D 14 (1976) 870.

[3] G.W. Gibbons, S.W. Hawking, Phys. Rev. D 15 (1977) 2738.

[4] N.D. Birrel, P.C.W. Davis, Quantum Fields in Curved Space, Cambridge Univ. Press, Cambridge, 1982.

[5] E.T. Akhmedov, D. Singleton, Int. J. Mod. Phys. A 22 (2007) 4797; E.T. Akhmedov, D. Singleton, arXiv: 0705.2525, JETP Lett., in press.

[6] P. Kraus, F. Wilczek, Nucl. Phys. B 437 (1995) 231; E. Keski-Vakkuri, P. Kraus, Nucl. Phys. B 491 (1997) 249.

[7] M.K. Parikh, F. Wilczek, Phys. Rev. Lett. 85 (2000) 5042; M.K. Parikh, Int. J. Mod. Phys. D 13 (2004) 2351.

[8] R. Brout, Ph. Spindel, Nucl. Phys. B 348 (1991) 405.

[9] T. Pilling, Phys. Lett. B 660 (2008) 402.

[10] J. Schwinger, Phys. Rev. 82 (1951) 664.

[11] E.T. Akhmedov, V. Akhmedova, D. Singleton, Phys. Lett. B 642 (2006) 124; E.T. Akhmedov, V. Akhmedova, D. Singleton, T. Pilling, Int. J. Mod. Phys. A 22 (2007) 1705.

[12] B.D. Chowdhury, Pramana 70 (2008) 593.

[13] E.T. Akhmedov, T. Pilling, D. Singleton, arXiv: 0805.2653 [gr-qc].

[14] G.E. Volovik, arXiv: 0803.3367. 